\title{
Comprendre la dynamique atmosphérique pour mieux reconstituer l'altitude passée des chaînes de montagnes
}

\author{
Svetlana Botsyun ${ }^{1}$, Pierre Sepulchre ${ }^{2}$, Camille Risi ${ }^{3}$ \\ 1. Department of Geosciences, University of Tübingen, Tübingen, Allemagne \\ 2. Laboratoire des sciences du climat et de l'environnement, IPSL, CEA / CNRS / \\ Université de Versailles Saint-Quentin-en-Yvelines / Université Paris-Saclay, \\ Gif-sur-Yvette \\ 3. Laboratoire de météorologie dynamique, IPSL, CNRS /Sorbonne Université, \\ Paris
}

svetlana.botsyun@uni-tuebingen.de

\section{Résumé}

Comprendre la dynamique de soulèvement d'une chaîne de montagne nécessite d'en estimer l'altitude passée. C'est le but de la paléoaltimétrie. L a méthodela plus répandue utilise la composition isotopique en oxygène des roches carbonatées formées dans les sols et à partir des sédiments lacustres. Celle-ci reflète la composition de la pluie passée qui, dans le monde actuel et dans la plupart des chaînes de montagnes, s'appauvrit progressivement en isotopes lourds avec l'altitude. En supposant que cet appauvrissement reste valide dans le passé, l'altitude du plateau tibétain à l'É ocène (il y a environ 42 millions d'années) est estimée à $4000 \mathrm{~m}$ environ. Mais d'autres marqueurs de l'altitude passée indiquent au contraire des altitudes inférieures à $2000 \mathrm{~m}$. La relation entre composition isotopique des pluies et altitude observée aujourd'hui s'applique-t-elle à l'É ocène? C'est ce que nous avons essayé de vérifier en utilisant un modèle de circulation générale atmosphérique, LM DZ-iso. On trouve qu'à l'É ocène la circulation atmosphérique et les processus hydrologiques étaient tellement différents de l'actuel que les observations isotopiques dans les roches carbonatées se trouvent finalement être cohérentesavec desaltitudes relativement
$\mathbf{R}$ econstituer l' histoire de la topographie de la surface terrestre est un prérequis fondamental pour de nombreuses disciplines des géosciences. Cela permet de comprendre les mécanismes à l'origine de la formation des chaînes de montagne (Fluteau, 2013), mais aussi de quantifier leur impact sur l'évolution du climat, notamment via les perturbations de la dynamique atmosphérique qu'impose la topographie (Broccoli et M anabe, 1992 ; K utzbach et al., 1993). II est donc essentiel de comprendre I'histoire de leur mise en place pour comprendre celle du climat et de la biodiversité.

La paléo-altimétrie est une estimation quantitative de l'altitude passée d'une surface par rapport au niveau moyen de la mer. Différentes techniques existent pour reconstruire les paléo-altitudes. Par exemple, la flore fossile permet d'estimer les paléoaltitudes, en supposant que la répartition des espèces végétales (obtenue par l'étude des grains de pollen fossiles) d'une part et la morphologie des feuilles (obtenue par l'étude de leurs fossiles) d'autre part sont un indice de l'altitude (Chaloner et Creber, 1990 ; M cElwain, 2004). D'autres techniques utilisent des marqueurs géologiques, comme les terrasses marines ou fluviales qui se forment lorsque l'al titude des montagnes par rapport au niveau de la mer varie de manière abrupte (G urrola et al., 2014). L'étude des taux de refroidissement des roches combinée aux simulations numériques des processus de surface (tectonique, érosion, incision par les rivières) (Ehlers et Farley, 2003), ainsi que, pour certains cas, la reconstruction des taux de sédimentation dans les bassins d'avant-pays (Potter et Szatmari, 2009) apportent aussi des informations indirectes sur l'altitude passée des montagnes. Enfin, l'analyse des isotopes stables de l'oxygène dans des archives géologiques permet aussi des reconstitutions quantitatives de l'altitude passée des montagnes, on parle alors de paléo-altimétrie isotopique. C'est aujourd' hui la méthode de reconstruction la plus utilisée et l'objet du présent article.

\section{Succès et limites de la paléo-altimétrie isotopique}

L'oxygène possédant plusieurs isotopes stables, il en résulte plusieurs formes de la molécule d'eau. Dans la nature, la forme la plus répandue est $\mathrm{H}_{2}{ }^{16} \mathrm{O}$ (>99,7\% des molécules), alors que $\mathrm{H}_{2}{ }^{18} \mathrm{O}$ ne représente qu'environ $0,2 \%$ des molécules. La proportion relative de ces différentes molécules (appelée rapport isotopique) est notée $\delta^{18} \mathrm{O}$ et est exprimée en pour mille (\%o) par rapport à une référence (un échantilIon d'eau marin). On peut mesurer ce 
faibles. Les différentes méthodes de paléo-altimétrie se retrouvent ainsi réconciliées et en accord avec un soulèvement récent (post-É ocène) du plateau tibétain.

\section{Abstract}

\section{Understanding atmospheric dynamics to better reconstruct past mountain elevation}

Understanding the uplift dynamics of a mountain range requires estimating past altitude. This is the purpose of the paleo-altimetry. T he most commonly applied paleo-altimetry method is based on the isotopic oxygen composition of the carbonate archives. It reflects the composition of past rain, which at present-day and in the most mountain ranges becomes progressively more depleted in heavy isotopes with altitude. Assuming that this depletion remains valid in the past, the elevation of the Tibetan Plateau in the E ocene (about 42 millions years ago) is estimated to be about $4000 \mathrm{~m}$. However, other proxy data indicate on the contrary low altitudes. Is the relationship between the rain isotopic composition and the altitude that is observed today applicable to the E ocene? This is what we tried to verify using an atmospheric general circulation model, LM DZ-iso. We find that in the E ocene, the atmospheric circulation and hydrological processes were so different to the present-day that the isotopic observations in the Eocene carbonates are actually consistent with relatively low altitudes of the Plateau. This allows us to reconcile different methods of paleo-altimetry in agreement with more recent (post-E ocene) uplift of the Tibetan Plateau. rapport isotopique dans les réservoirs d'eau actuels (précipitation, vapeur d'eau, océan, eau souterraine, rivière, glace) (Risi et Bony, 2011 ; V imeux, 2013), mais aussi dans les roches carbonatées telles que les spéléothèmes, les carbonates présents dans les sols (figure 1) ou d'origine lacustre. En effet, la formation de ces carbonates repose sur l'utilisation de l'oxygène de l'eau de pluie. Un fractionnement isotopique, qui dépend de la température des sols, a lieu entre l'eau de pluie et les carbonates lors de ce processus chimique. L'évaporation de l'eau après précipitation (Haese et al., 2013 ; Risi et al., 2016) et les processus de diagenèse (Garzione et al., 2004) sont susceptibles de perturber la composition des roches. Mais, en première approximation, les variations de la composition isotopique dans les archives carbonatées reflètent celles existant dans les paléo-précipitations (Rowley et $\mathrm{G}$ arzione, 2007).

Les reconstructions paléo-altimétriques fondées sur l'analyse des isotopes stables de l'oxygène reposent sur l'évolution du $\delta^{18} O$ des précipitations $\left(\delta^{18} \mathrm{O}_{\mathrm{p}}\right)$ en fonction de la température, selon le modèle de la distillation de Rayleigh. Prenons une masse d'air avec de la vapeur d'eau fraîchement évaporée de l'océan; lorsque l'air s'élève le long des flancs d'une chaîne de montagnes, elle se refroidit et une partie de la vapeur d'eau se condense dans les nuages. Les molécules d'eau contenant les isotopes les plus lourds se condensent de manière préférentielle, un processus appelé fractionnement isotopique. L'eau qui précipite est donc enrichie en ${ }^{18} \mathrm{O}$ et la valeur de $\delta^{18} \mathrm{O}$ augmente. A u contraire, la vapeur résiduelle se retrouve appauvrie ${ }^{18} 0$. A u fur et à mesure que l'air s'élève en altitude, les précipitations deviennent de plus en plus pauvres en isotopes lourds (figure 2). À l'époque actuelle, le rapport isotopique $\delta^{18} \mathrm{O}_{p}$ diminue avec l'altitude selon un gradient moyen global de 2,8\% $\mathrm{km}^{-1}$ (Quade et al., 2011).
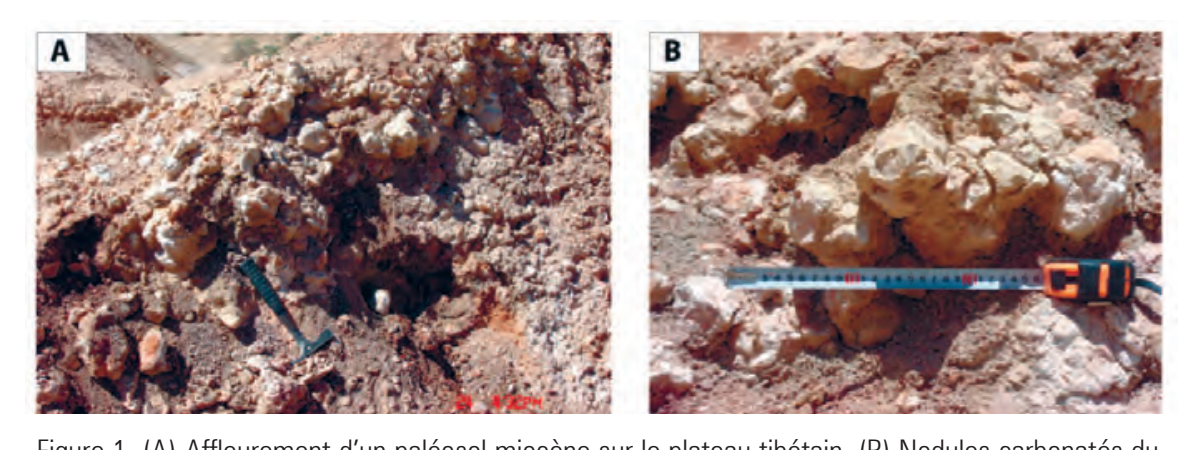

Figure 1. (A) Affleurement d'un paléosol miocène sur le plateau tibétain. (B) Nodules carbonatés du

même paléosol. Photos de Guangsheng Zhuang.
Plusieurs conditions sont cependant nécessaires pour obtenir des paléoaltitudes à partir des isotopes de l'eau : - le $\delta^{18} 0$ doit être mesuré dans des archives carbonatées $\left(\delta^{18} O_{c}\right)$;

- le $\delta^{18} \mathrm{O}$ des paléoprécipitations $\left(\delta^{18} \mathrm{O}_{\mathrm{p}}\right)$ peut être estimé à partir $\mathrm{du}$ $\delta^{18} \mathrm{O}_{c}$ en tenant compte de l'équilibre entre l'eau et la calcite en fonction de la température ;

- le $\delta^{18} O_{p}$ est converti en paléo-altitude en utilisant des relations théoriques entre $\delta^{18} O_{p}$ et al titude.

A ujourd'hui, la relation théorique entre $\delta^{18} O_{p}$ et altitude la plus largement utilisée pour les reconstructions paléo-altimétriques repose sur un modèle thermodynamique unidimensionnel (Rowley et al., 2001). $\mathrm{Ce}$ modèle considère une parcelle d'air ascendante isolée avec une température initiale prescrite $\left(T_{0}\right)$, une humidité relative $\left(R h_{0}\right)$ et une valeur de référence pour la composition de la vapeur d'eau avant le processus de précipitation $\left(\delta^{18} \mathrm{O} \vee 0\right)$. En tenant compte de l'ascendance adiabatique de l'air et de la distillation de R ayleigh de la vapeur d'eau, le $\delta^{18} 0$ des précipitations en fonction de l'altitude peut être approximé avec un polynôme de second degré. Malgré le succès et la validation de ce modèle dans plusieurs régions montagneuses (Rowley et Garzione, 2007), son utilisation dans un contexte paléoclimatique est confrontée à plusieurs limites.

Tout d'abord, le choix de valeurs de référence uniques pour $T_{0}, R h_{0}$ et $\delta^{18} \mathrm{O}_{\mathrm{v}}$ repose nécessairement sur des approximations fortes. À l'échelle géologique, les variations de la concentration en $\mathrm{CO}_{2}$ atmosphérique, les changements de disposition des masses continentales en lien avec la tectonique des plaques et les variations d'insolation affectent le cycle hydrologique, la circulation atmosphérique, I'humidité relative et donc in fine les valeurs de $\delta^{18} \mathrm{O}_{\mathrm{p}}$. Ensuite, à l'échelle régionale, la présence d'un massif montagneux altère également les diffé- 

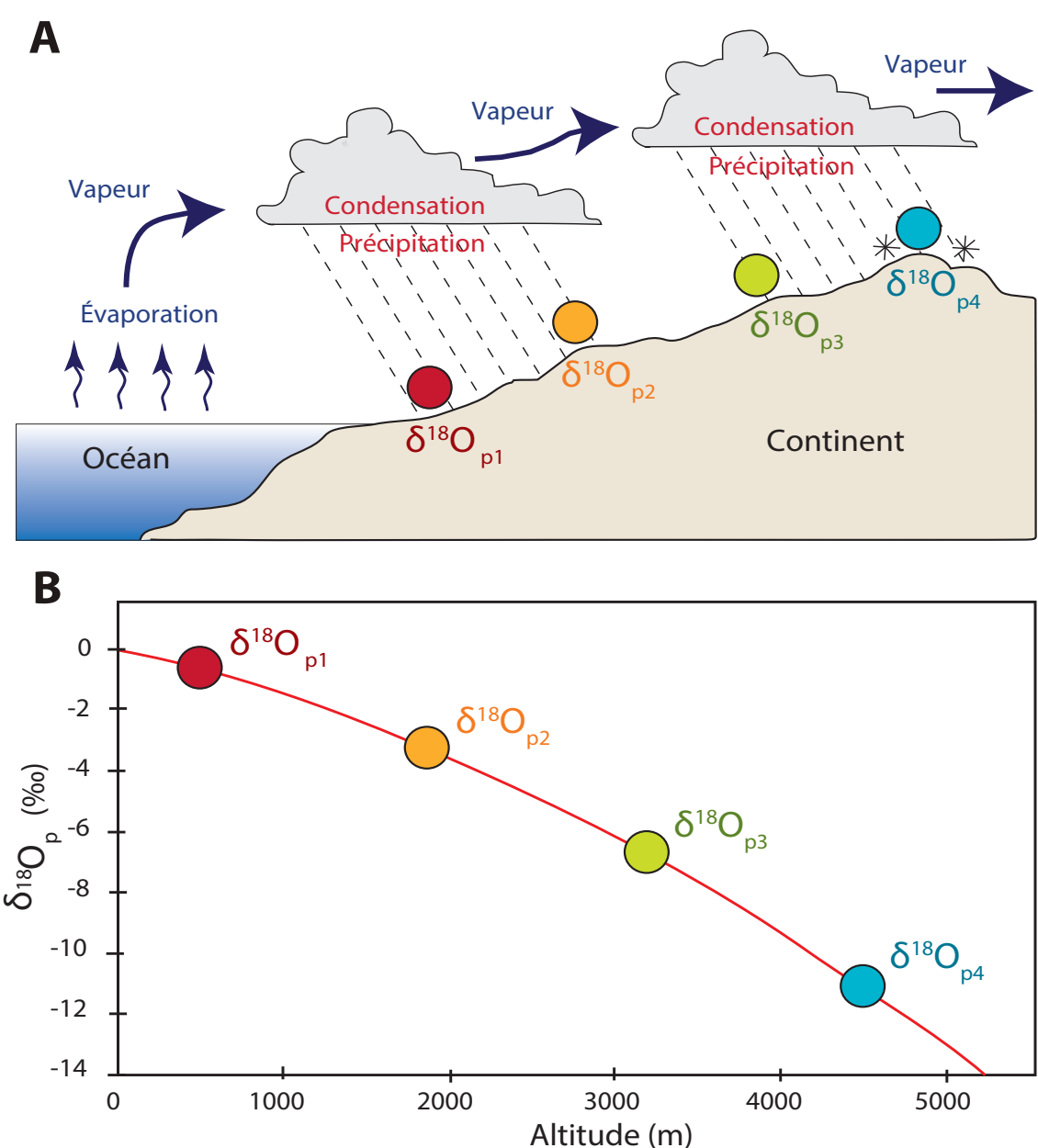

Figure 2. (A) Schéma de la distillation de Rayleigh. (B) Rapport isotopique $\delta^{18} \mathrm{O}$ en fonction de l'altitude.

rents paramètres déterminants pour le $\delta^{18} \mathrm{O}_{\mathrm{p}}$, comme nous l'avons montré avec de simples expériences de sensibilité à la topographie (Botsyun et al., 2016) réalisées avec un modèle de circulation générale qui inclut une représentation des isotopes de l'eau, LM DZ-iso (Risi et al., 2010). Nous avons donc cherché à dépasser le modèle classique en simulant de manière explicite la composition isotopique de I'eau et tous les paramètres cl imatiques qui la contrôlent, dont la circulation atmosphérique, dans un contexte paléoclimatique. Pour cela, nous avons utilisé L M DZ-iso et nous nous sommes intéressés à la paléo-altitude du plateau tibétain à l'É ocène, il y a environ 42 millions d'années.

\section{L'exemple du plateau tibétain à l'Éocène}

La collision de l'Inde avec la marge sud de l'Eurasie, il y a environ 50 millions d'années $(\mathrm{M} a)$, est à l'origine du soulèvement de l'Himalaya et du plateau tibétain. La barrière orographique ainsi formée contrôle la circulation atmosphérique en A sie. Cette région est considérée comme un véritable "laboratoire naturel » pour l'étude des interactions entre tectonique et climat, car les observations de terrain ainsi que les simulations numériques suggèrent un impact important de la surrection de l'Himalaya et du plateau tibétain sur les changements climatiques à différentes échelles de temps.

La paléo-altimétrie isotopique a été appliquée à la chaîne de l'H imalaya et au plateau tibétain pour les périodes de temps allant du Paléocène au Pléistocène $(-60$ à -2 millions d'années). Pour l'Éocène (-56 à -34 millions d'années), les différentes estimations paléo-altimétriques sont contradictoires: la paléo-altimétrie isotopique suggère des altitudes proches des valeurs actuelles (>4 $000 \mathrm{~m}$ ) (Rowley et Currie, 2006 ; $X u$ et al., 2013; Ding et al., 2014), alors que les données paléobotaniques (Song et al., 2010; Wang et al., 2018) et paléontologiques (Wei et al., 2016; Wu et al., 2017) suggèrent des altitudes beaucoup plus basses $(0-2000 \mathrm{~m})$.
Ce désaccord pourrait-il être lié aux limitations de la paléo-altimétrie isotopique citées plus haut?

\section{Les simulations avec LMDZ-iso}

L'utilisation d'une grille conçue spécifiquement pour cette étude, bénéficiant d'un zoom sur l'A sie du Sud-Est, a permis d'atteindre une résolution spatiale moyenne de l'ordre de $50 \mathrm{~km}$ sur l'A sie centrale, au prix d'une résolution plus grossière sur le reste du globe, ce qui fournit un bon compromis entre un temps de calcul raisonnable et une résolution spatiale permettant de représenter correctement les caractéristiques principales de la topographie du plateau tibétain. Avec cette configuration, LM DZ-iso est capable de si muler de façon satisfaisante les répartitions spatiales de $\delta^{18} \mathrm{O}_{p}$ (figure $3, A$ ), la température (figure 3 , $\mathrm{G)}$, la circulation atmosphérique et la quantité de précipitation (figure $3, \mathrm{~K}$ ) pour le climat actuel (avec orographie, géographie, concentrations en $\mathrm{CO}_{2}$ et conditions océaniques de surface actuelles).

A fin d'étudier comment l'altitude du plateau tibétain influence la composition isotopique des carbonates, nous avons réalisé une série de simulations pour différentes altitudes du plateau tibétain dans le contexte paléogéographique de l'É ocène. La reconstruction paléogéographique globale de l'É ocène (Licht et al., 2014) est issue de l'utilisation combinée de modèles globaux de tectonique des plaques et de reconstructions paléogéographiques. À I'Éocène, le sous-continent indien était positionné plus au sud et une mer épicontinentale (la Paratéthys) occupait une partie de l'Eurasie. Nous avons réalisé une simulation avec un plateau tibétain à $5000 \mathrm{~m}$ (figure 3, EOC-X L), c'est-à-dire une à altitude comparable à l'altitude actuelle, et une autre avec un plateau tibétain à $200 \mathrm{~m}$ (figure 3 , EOC-S).

Nous avons appliqué une concentration en $\mathrm{CO}_{2}$ atmosphérique de $1120 \mathrm{ppm}$, cohérente avec les reconstitutions de l'époque. LMDZ-iso a été forcé aux limites par des températures océaniques de surface issues d'un modèle couplé océanatmosphère (FOAM, Jacob, 1997), ainsi que la répartition de la végétation simulée avec un modèle dédié (LP), Sitch et al., 2003). 

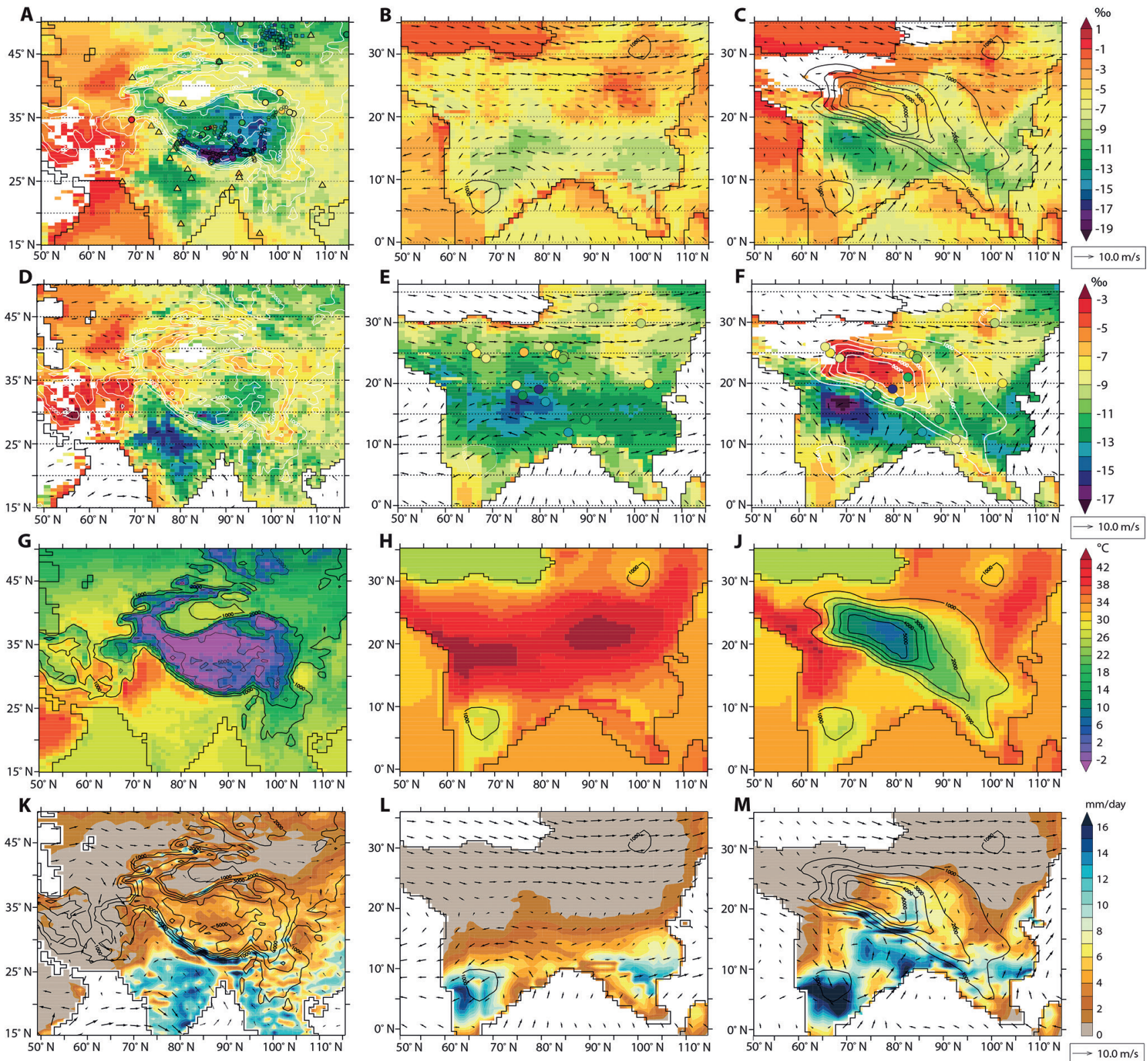

Figure 3. $(A, B, C)$ Rapport isotopique $\delta^{18} \mathrm{O}$ des précipitations pour les régions où la précipitation est supérieure à $0,1 \mathrm{~mm} / \mathrm{jour}$. (D, E, F) Rapport isotopique $\delta^{18} \mathrm{O}$ de la calcite sur le continent. $(\mathrm{G}, \mathrm{H}, \mathrm{J})$ Température de surface et $(\mathrm{K}, \mathrm{L}, \mathrm{M})$ quantité de précipitation sur le continent en été (de mai à septembre) simulés par LMDZ-iso pour (A, D, G, K) l'époque actuelle et à l'Éocène pour deux scénarios extrêmes d'altitude : $(B, E, H, L)$ avec un Tibet bas (EOC-S) et $(C, F, J, M)$ avec un Tibet haut (EOC-XL). Les flèches sur les figures correspondent aux vents de la basse troposphère.

\section{Résultats des simulations}

L'augmentation $\mathrm{du}$ forçage radiatif liée à la forte teneur en $\mathrm{pCO}_{2}$ atmosphérique utilisée dans nos simulations de l'Éocène (simulations EOC-S et EOC-XL) implique une réorganisation de la dynamique atmosphérique à grande échelle. On se concentre ici sur les résultats pour l'été (de mai à septembre), car c'est principalement lors de cette saison que les roches carbonatées se forment (Quade et al., 2007). Dans l'hémisphère Nord, les vents d'ouest de moyennes latitudes sont en moyenne plus intenses à la surface que dans la simulation pour la période actuelle. Les simulations de l'Éocène sont également caractérisées par une forte subsidence atmosphérique sur le continent asiatique dans les moyennes latitudes. Le cycle hydrologique du sous-continent indien est caractérisé par des précipitations dont la saisonnalité est semblable à celle de la mousson actuelle, mais dont l'intensité est plus élevée (figure 3, $\mathrm{K}, \mathrm{L}, \mathrm{M})$. En revanche, sur la région du plateau tibétain et sur l'Asie centrale, l'interaction des vents d'ouest renforcés et de la subsidence atmosphérique à grande échelle provoque une aridité étendue. Les fortes précipitations sur le sous-continent indien diminuent le rapport isotopique $\delta^{18} \mathrm{O}_{\mathrm{p}}$ de la pointe sud de l'Inde (d'environ $4 \%$ ) à un minimum situé à $15^{\circ} \mathrm{N}$ sur l'avant-pays indien (de -8 à $-11 \%$, en fonction de la topographie). Ceci s'explique par un appauvrissement progressif en $\delta^{18} \mathrm{O}$ de la vapeur d'eau par la convection atmosphérique (Risi et Bony, 2011). $\mathrm{Au}$ contraire, les environnements arides $(<0,5 \mathrm{~mm} /$ jour $)$ sur le nord du plateau tibétain et en Asie centrale sont associés à des valeurs de $\delta^{18} \mathrm{O}_{\mathrm{p}}$ beaucoup plus élevées (de -3 à $-1 \%$ ).

Le résultat le plus surprenant et contre-intuitif de nos simulations à l'Éocène est donc un gradient isotopique inversé sur le flanc sud du plateau tibétain, avec des valeurs de $\delta^{18} \mathrm{O}_{\mathrm{p}}$ allant de $-8 \%$ au pied du plateau tibétain à $-5 \%$ au sommet du flanc sud (figure 4, C). Un tel gradient est en contradiction avec les valeurs modernes observées et simulées qui décrivent une diminution du rapport $\delta^{18} \mathrm{O}$ de -8 à $-18 \%$ à mesure que l'altitude augmente sur le flanc sud du plateau tibétain (figure 4, A). 

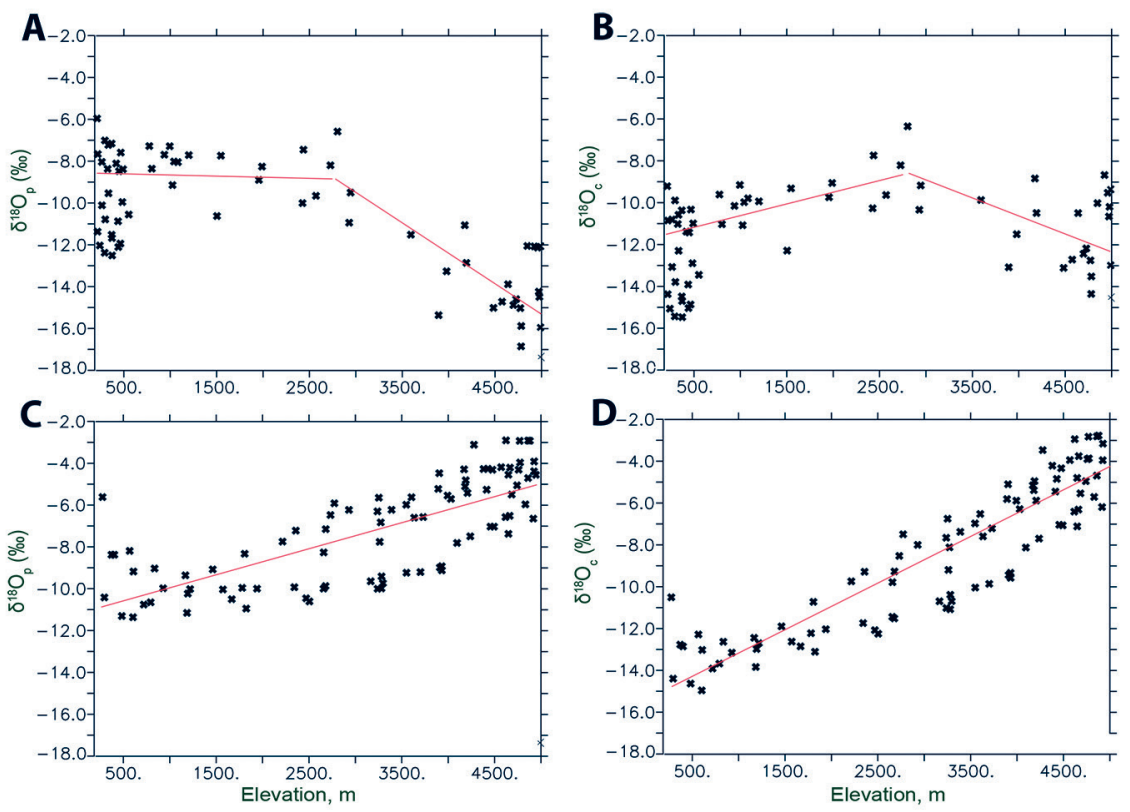

Figure 4. (A, C) Rapports isotopiques $\delta^{18} \mathrm{O}_{p}$ des précipitations. (B, D) $\delta^{18} \mathrm{O}_{c}$ des roches carbonatées simulés par LMDZ-iso en fonction de l'altitude: (A, B) à travers l'Himalaya aujourd'hui (gradient isotopique normal) et $(C, D)$ dans la partie sud du plateau tibétain élevé à l'Éocène (EOC-XL). On observe un gradient isotopique inversé.

Plusieurs raisons sont susceptibles d'expliquer ce changement de gradient isotopique par rapport à l'époque actuelle (figure 5).

L'Inde, environ $10^{\circ}(1100 \mathrm{~km})$ plus au sud, ainsi que la concentration élevée en $\mathrm{CO}_{2}$ atmosphérique à l'Éocène, induisent un climat beaucoup plus chaud que l'actuel sur cette région. Alors que de nos jours les précipitations le long des trajectoires montant vers l'Himalaya sont principalement d'origine orographique, dans nos simulations à l'Éocène (EOC-S et EOC-XL) celles montant sur le proto-Himalaya sont principalement d'origine convective. Or les processus menant aux précipitations orographiques appauvrissent beaucoup plus efficacement en ${ }^{18} \mathrm{O}$ l'eau atmosphérique que les processus de convection. En effet, lors de la convection, l'appauvrissement en ${ }^{18} \mathrm{O}$ par la condensation est partiellement compensé par l'apport de vapeur enrichie en ${ }^{18} \mathrm{O}$ par les panaches ascendants.
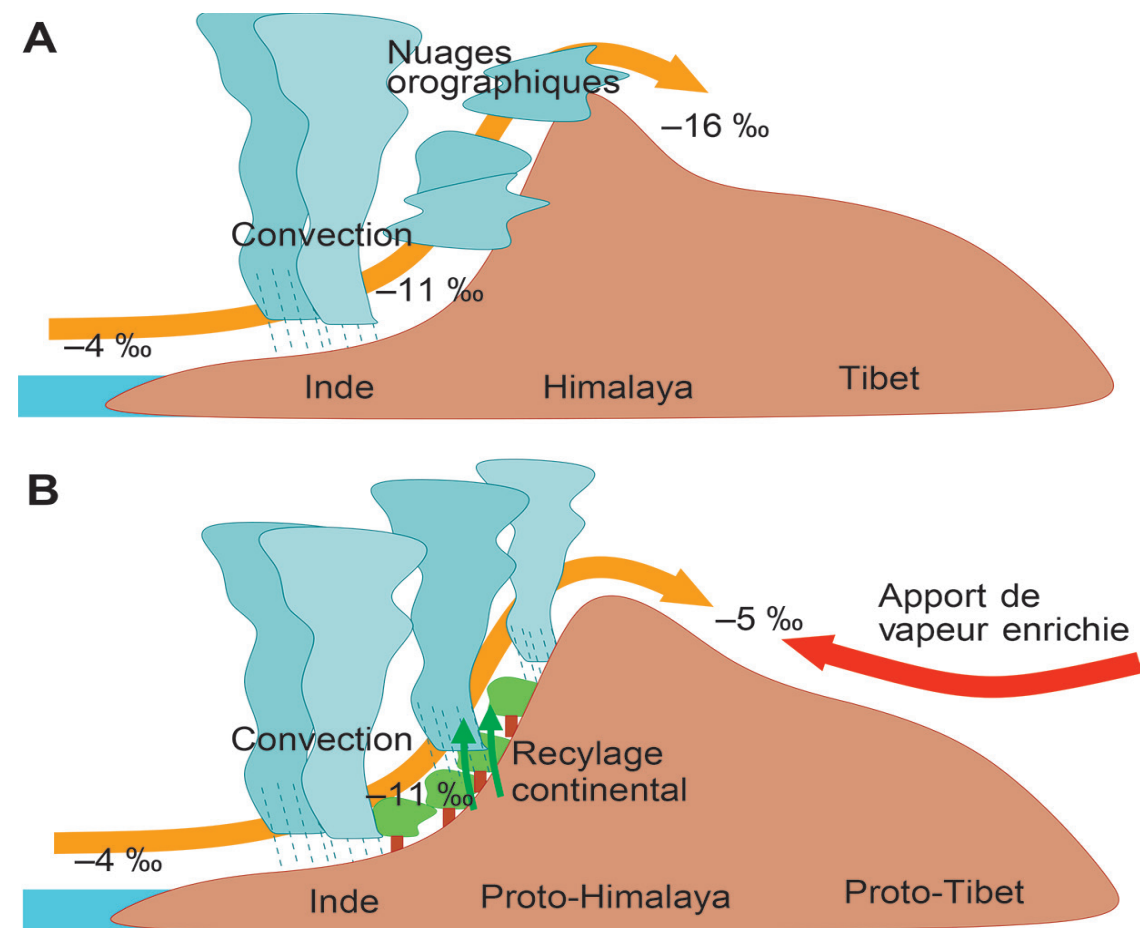

Figure 5. Schéma des processus atmosphériques contrôlant le $\delta^{18} \mathrm{O}$ de la précipitation au Tibet : (A) à l'époque actuelle ; (B) à l'Éocène dans le cas d'un haut plateau tibétain.

Une part importante de la vapeur d'eau au-dessus du plateau tibétain à l'Éocène est apportée par des masses d'air venant d'autres régions, notamment du nord-ouest, où elles se rechargent en vapeur d'eau et en ${ }^{18} \mathrm{O}$ au-dessus de la Paratéthys (figure 5). Un modèle de distillation de Rayleigh le long d'une trajectoire unique ne semble donc pas applicable dans le cas de l'Éocène.

Ceci explique l'origine du gradient isotopique inversé simulé par LMDZiso à l'Éocène et met en évidence les limitations des estimations paléoaltimétriques basées sur le gradient isotopique actuel.

Ces simulations numériques fournissent également une occasion de réinterpréter les valeurs de $\delta^{18} \mathrm{O}_{\mathrm{p}}$ déduits des archives géologiques. En utilisant les valeurs simulées de $\delta^{18} \mathrm{O}_{\mathrm{p}}$ et de température du sol en été, nous avons pu recalculer les valeurs de $\delta^{18} \mathrm{O}_{c}$ pour différents scénarios d'altitude et les comparer aux mesures issues des archives géologiques (figure 3, E, F). Les analyses statistiques montrent que le scénario d'un plateau tibétain bas (moins de $2000 \mathrm{~m}$ ) est celui qui produit le meilleur accord avec les observations, suggérant donc qu'à l'Éocène le proto-Himalaya et le plateau tibétain n'avaient pas encore atteint leur altitude actuelle.

\section{Conclusions}

Nos simulations suggèrent une relation entre la topographie et la composition isotopique de la précipitation plus complexe que celle décrite par des modèles basés sur la seule distillation de Rayleigh. Les études précédentes basées sur une telle relation suggèrent que le plateau tibétain était déjà élevé (plus de $4000 \mathrm{~m}$ ) à l'Éocène. Au contraire, nos simulations, qui prennent en compte la réponse de la dynamique atmosphérique et du fractionnement isotopique aux forçages de l'Éocène (paléogéographie, $\mathrm{CO}_{2}$ atmosphérique plus élevé) montrent que les valeurs de $\delta^{18} \mathrm{O}_{c}$ observées sont plus cohérentes avec des altitudes plus modestes. En outre, ces nouvelles estimations réconcilient la paléo-altimétrie isotopique avec les reconstructions issues des données fossiles (Song et al., 2010 ; Wei et al., 2016; Wu et al., 2017 ; Wang et al., 2018). Notre étude montre à quel point la compréhension de la dynamique atmosphérique et de ses conséquences sur la composition isotopique de l'eau, 
par le biais de simulations avec un modèle de circulation générale, est un prérequis pour interpréter correctement les archives géologiques et pour reconstituer l'histoire des chaînes de montagnes et des hauts plateaux. Ces résultats ont toutefois été obtenus avec un seul modèle: sont-ils robustes?
Sont-ils sensibles à la façon dont LMDZ-iso représente les processus atmosphériques ? Pour répondre à ces questions, il serait utile de reproduire les mêmes simulations avec d'autres modèles de circulation générale, dans le cadre d'un projet d'intercomparaison.

\section{Remerciements}

Cet article est basé sur un article publié dans Science (Botsyun et al., 2019). N ous remercions les autres coauteurs de l'article original : Yannick Donnadieu, A lexis Licht et Jeremy K. Caves Rugenstein.

\section{Bibliographie}

Botsyun S., Sepulchre P., Risi C., Donnadieu Y., 2016. Impacts of Tibetan Plateau uplift on atmospheric dynamics and associated precipitation $\delta^{18} \mathrm{O}$. Clim. Past, $12,1401-1420$. doi: 10.5194/cp-12-1401-2016

Botsyun S., Sepulchre P., Donnadieu Y., Risi C., Licht A., Caves Rugenstein J.K., 2019. Revised paleoaltimetry data show low Tibetan Plateau elevation during the Eocene. Science, 363. doi: 10.1126/science.aaq1436

Broccoli A.J., Manabe S., 1992. The effects of orography on midlatitude Northern Hemisphere dry climates. J. Clim., 5, $1181-1201$.

Chaloner W., Creber G., 1990. Do fossil plants give a climatic signal? J. Geol. Soc., 147, 343-350.

Ding L., Xu Q., Yue Y., Wang H., Cai F., Li S., 2014. The Andean-type Gangdese Mountains: Paleoelevation record from the Paleocene-Eocene Linzhou Basin. Earth Planet. Sci. Lett., 392, 250-264. doi: 10.1016/j.epsl.2014.01.045

Ehlers T.A., Farley K.A., 2003. Apatite (U-Th)/He thermochronometry: methods and applications to problems in tectonic and surface processes. Earth Planet. Sci. Lett., 206, 1-14. doi: 10.1016/S0012-821X(02)01069-5

Fluteau F., 2013. L'évolution des climats à l'échelle des temps géologiques, le rôle des changements paléogéographiques. In : Paléoclimatologie, Enquête sur les climats anciens, J.-C. Duplessy et G. Ramstein (Eds), EDP Sciences, pp. 77-136.

Garzione C.N., Dettman D.L., Horton B.K., 2004. Carbonate oxygen isotope paleoaltimetry: evaluating the effect of diagenesis on paleoelevation estimates for the Tibetan plateau. Palaeogeogr. Palaeoclim. Palaeoecol., 212, 119-140.

Gurrola L.D., Keller E.A., Chen J.H., Owen L.A., Spencer J.O., 2014. Tectonic geomorphology of marine terraces; Santa Barbara fold belt, California. Geol. Soc. Am. Bull., 126, 219-233. doi: 10.1130/B30211.1

Haese B., Werner M., Lohmann G., 2013. Stable water isotopes in the coupled atmosphere-land surface model ECHAM5-JSBACH. Geosci. Model Dev., 6, 1463-1480.

Jacob R.L., 1997. Low frequency variability in a simulated atmosphere ocean system. Doctoral dissertation, University of Wisconsin Madison. Kutzbach J.E., Prell W.L., Ruddiman W.F., 1993. Sensitivity of Eurasian climate to surface uplift of the Tibetan Plateau. J. Geol., 101, 177-190.

Licht A., van Cappelle M., Abels H.A., Ladant J.-B., Trabucho-Alexandre J., France-Lanord C, Donnadieu Y., Vandenberghe J., Rigaudier T., Lécuyer C., Terry Jr D., Adriaens R., Boura A., Guo Z., Aung Naing S., Quade J., Dupont-Nivet G, Jaeger J.-J., 2014. Asian monsoons in a late Eocene greenhouse world. Nature, 513, $501-506$.

McElwain J.C., 2004. Climate-independent paleoaltimetry using stomatal density in fossil leaves as a proxy for $\mathrm{CO}_{2}$ partial pressure. Geology, 32, 1017. doi: 10.1130/G20915.1 Potter P.E., Szatmari P., 2009. Global Miocene tectonics and the modern world. Earth Sci. Rev., 96, 279-295.

Quade J., Garzione C., Eiler J., 2007. Paleoelevation reconstruction using pedogenic carbonates. Rev. Mineral. Geochem., 66, 53-87.

Quade J., Breecker D.O., Daëron M., Eiler J., 2011. The paleoaltimetry of Tibet: An isotopic perspective. Am. J. Sci., 311, 77-115.

Risi C., Bony S., 2011. Évaluer les modèles de climat et leurs projections : la valeur ajoutée de la composition isotopique de l'eau. La Météorologie, 74, 52-51.

Risi C., Bony S., Vimeux F., Jouzel J., 2010. Water-stable isotopes in the LMDZ4 general circulation model: Model evaluation for present-day and past climates and applications to climatic interpretations of tropical isotopic records. J. Geophys. Res. Atmos., 115, 1-27. doi: 10.1029/2009JD013255

Risi C., Ogée J., Bony S., Bariac T., Raz-Yaseef N., Wingate L., Welker J., Knohl A., Kurz-Besson C., Leclerc M., Zhang G., Buchmann N., Santrucek J., Hronkova M., David T.S., Peylin P., Guglielmo F., 2016. The water isotopic version of the land-surface model ORCHIDEE: Implementation, evaluation, sensitivity to hydrological parameters. Hydrol. Curr. Res., 7, 1000258. doi: 10.4172/2157-7587.1000258

Rowley D.B., Currie B.S., 2006. Palaeo-altimetry of the late Eocene to Miocene Lunpola basin, central Tibet. Nature, 439, 677-681. doi: 10.1038/nature04506 Rowley D.B., Garzione C.N., 2007. Stable isotope-based paleoaltimetry. Ann. Rev. Earth Planet. Sci., 35, 463-508. doi: 10.1146/annurev.earth.35.031306.140155

Rowley D.B., Pierrehumbert R.T., Currie B.S., 2001. A new approach to stable isotope-based paleoaltimetry: Implications for paleoaltimetry and paleohypsometry of the High Himalaya since the late Miocene. Earth Planet. Sci. Lett., 188, 253268. doi: 10.1016/S0012-821X(01)00324-7

Sitch S., Smith B., Prentice I.C., Arneth A., Bondeau A., Cramer W., Kaplan J.O., Levis S., Lucht W., Sykes M.T., Thonicke K., Venesky S., 2003. Evaluation of ecosystem dynamics, plant geography and terrestrial carbon cycling in the LPJ dynamic global vegetation model. Global Change Biol., 9, 161-185.

Song X.Y., Spicer R.A., Yang J., Yao Y.F., Li C.S., 2010. Pollen evidence for an Eocene to Miocene elevation of central southern Tibet predating the rise of the High Himalaya. Palaeogeogr. Palaeoclim. Palaeoecol., 297, 159-168. doi: 10.1016/j.palaeo.2010.07.025

Vimeux F., 2013. La composition isotopique de la vapeur d'eau ; apport à l'étude des processus atmosphériques. La Météorologie, 81, 31-40.

Wang H., Dutta S., Kelly R.S., Rudra A., Li S., Zhang Q.-0., Zhang Q.-0., Wu Y.-X., Cao M.-Z., Wang B., 2018. Amber fossils reveal the Early Cenozoic dipterocarp rainforest in central Tibet. Palaeoworld, 27, 506-513.

Wei Y., Zhang K., Garzione C.N., Xu Y., Song B., Ji J., 2016. Low palaeoelevation of the northern Lhasa terrane during late Eocene: Fossil foraminifera and stable isotope evidence from the Gerze Basin. Sci. Reports, 6, 27508

Wu F., Miao D., Mann Chang M., Shi G., Wang N., 2017. Fossil climbing perch and associated plant megafossils indicate a warm and wet central Tibet during the late Oligocene. Sci. Reports, 7, 1-7. doi: 10.1038/s41598-017-00928-9

Xu O., Ding L., Zhang L., Cai F., Lai O., Yang D., Liu-Zeng J., 2013. Paleogene high elevations in the Qiangtang Terrane, central Tibetan Plateau. Earth Planet. Sci. Lett., 362, 31-42. doi: 10.1016/j.epsl.2012.11.058 